\title{
Five plus two equals yellow
}

\section{Mental arithmetic in people with synaesthesia is not coloured by visual experience.}

n synaesthesia, ordinary stimuli elicit extraordinary experiences. For example when C., who is a digit-colour synaesthete, views black digits, each number elicits a photism - a visual experience of a specific colour. It has been proposed that synaesthetic experiences differ from imagery in their consistency ${ }^{1}$, automaticity ${ }^{2,3}$ and reliance on external stimuli to induce them ${ }^{4}$. Here we demonstrate that C.'s photisms are both consistent and automatic, but we find that an externally presented inducing stimulus is not necessary to trigger a photism and that simply activating the concept of a digit is sufficient.

As a measure of consistency, we asked C. to name the colour of her photisms elicited by the digits 1 to 9 shown in random order, 10 times each. C.'s pairings between digits and colour names were $100 \%$ consistent across repetitions.

To assess automaticity, we used a Stroop task. Colour-naming reaction times were recorded for coloured squares (baseline condition) or for digits displayed in colours that were either congruent or incongruent with C.'s photisms (Fig. 1). Her mean reaction times for each condition were: congruent, $552 \mathrm{~ms}$ (error, 1.4\%); baseline, $545 \mathrm{~ms}$ (error, $0.0 \%$ ); incongruent, $797 \mathrm{~ms}$ (error, $2.8 \%)$. An analysis of variance $(F(2,410)=$ 74.88, $P<0.001)$ and Tukey post-hoc analyses revealed that incongruent trial reaction times were significantly slower than baseline $(P<0.001)$ and congruent trial reaction times $(P<0.001)$ - the last two conditions did not differ.

C.'s large significant difference in congruent/incongruent reaction times (245 $\mathrm{ms})$ contrasts with the small, non-significant, congruent/incongruent differences (ranging from -11 to $17 \mathrm{~ms}$ ) of eight nonsynaesthetes tested using identical stimuli. Figure 1a shows the Stroop performance of $\mathrm{C}$. and of the non-synaesthete most similar to C. in terms of baseline-trial colour-naming reaction times. C.'s performance indicates that the digits automatically elicited photisms that interfered with her colour-naming on incongruent trials.

For C., viewing the digit 7 automatically induces a yellow photism. To assess whether C.'s photisms could be elicited without physically showing her an inducing stimulus, we presented, in sequence, a digit (for example, 4), an operator (for example, + ), a second digit (for example, 3 ), and a colour patch. C. had to name the colour patch as quickly as possible, and then report the solution to the arithmetic problem. On each trial, the colour patch was either

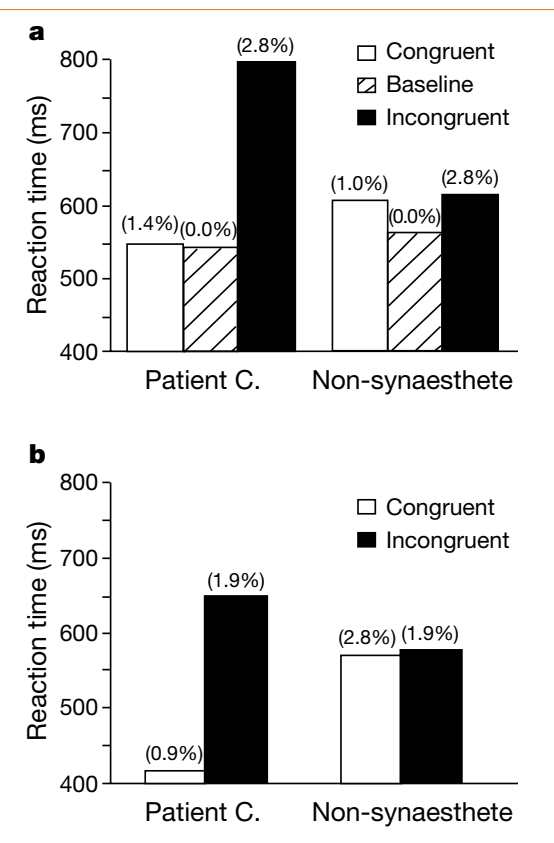

congruent or incongruent with the photism associated with the arithmetic solution. For example, $5+2$ was followed either by a yellow patch, which is congruent with C.'s photism colour for 7 , or a red patch, which is incongruent with C.'s photism for 7 . To discourage $\mathrm{C}$. from using the arithmetic solution to predict the colour of the patch, we presented three times as many incongruent trials as congruent trials.

If automatic photisms can be induced simply by calculating the solution to arithmetic problems, then they should interfere with C.'s ability to name the colour of the patch on incongruent trials but not on congruent trials. Consistent with our reasoning, C.'s colour-naming reaction times were significantly slower on incongruent trials (mean, $650 \mathrm{~ms}$ ) than on congruent trials (mean, $414 \mathrm{~ms}$ ), $t(390)=$ 19.48, $P<0.001$. This large congruent/ incongruent difference (236 ms) was 19.3 standard deviations away from the mean difference (1 ms) obtained for eight nonsynaesthetes. Figure 1b shows C.'s performance compared with the performance of the non-synaesthete who was most similar to C. in terms of overall reaction times.

C.'s large difference in congruent/incongruent reaction times $(236 \mathrm{~ms})$ indicates that automatic photisms were induced by the arithmetic solution (for example, the yellow photism associated with the digit 7 was generated in response to calculating $5+2$ ). This suggests that an external stimulus (for example, a physically present numeral 7) is not required to trigger a
Figure 1 Colour-naming reaction times (and errors) for a digit-colour synaesthete, C., and for representative non-synaesthetes. a, Digit Stroop task: on each trial, reaction times were recorded for naming coloured squares ('baseline') or digits displayed in colours that were either congruent or incongruent with C.'s photism colours. There were 144 trials of each type, randomly intermixed. b, Arithmetic Stroop task: on each trial, participants were presented, in sequence, with a fixation cross, a digit, an arithmetic operator, a second digit, and a colour patch. Participants named the colour patch as quickly as possible and then reported the arithmetic solution. Colour patches were either congruent (108 trials) or incongruent (324 trials) with C.'s photism colours for the arithmetic solutions. Trial types were intermixed. Further details are available from the authors.

photism. Rather, activating the concept of a digit by a mental calculation was sufficient to induce a colour experience. Thus, although C.'s photisms are both consistent and automatic, they do not require a physical inducing stimulus to elicit them.

Stroop-type paradigms have shown that photisms are automatic and involuntary ${ }^{4}$. Brain imaging has demonstrated that photisms activate visual-association areas involving binding colour to form ${ }^{5}$. Cognitive research, brain imaging and even genetics (synaesthesia runs in families) ${ }^{6}$ all attest to the reality of synaesthesia. Although the neural architecture and neurodevelopmental aspects of synaesthesia are not yet fully understood, the finding that photisms can be concept-driven may help to guide our understanding of this rare phenomenon.

Mike J. Dixon, Daniel Smilek, Cera Cudahy, Philip M. Merikle

Department of Psychology, University of Waterloo,

Waterloo, Ontario N2L 3G1, Canada

e-mail:mjdixon@watarts.uwaterloo.ca

Baron-Cohen, S., Wyke, M. \& Binnie, C. Perception 16, 761-767 (1987).

2. Mills, C. B., Boteler, E. H. \& Oliver, G. K. Cogn. Neuropsychol. 16, 181-191 (1999).

3. Odgaard, E. C., Flowers, J. H. \& Bradman, H. L. Perception 28, 651-664 (1999).

4. Cytowic, R. E. The Man who Tasted Shapes (Putnam, New York, 1993).

5. Frith, C. D. \& Paulesu, E. in Synaesthesia: Classic and Contemporary Readings (eds Baron-Cohen, S. \& Harrison, J. E.) 123-147 (Blackwell, Cambridge, Massachusetts, 1997).

6. Bailey, M. E. S. \& Johnson, K. J. in Synaesthesia: Classic and Contemporary Readings (eds Baron-Cohen, S. \& Harrison, J. E.) 182-210 (Blackwell, Cambridge, Massachusetts, 1997). 